\title{
Evidence of Pressure Waves in Confined Laser Ablation
}

\author{
Matthias DOMKE ${ }^{* 1}$, Dominik FELSL ${ }^{* 2}$, Stephan RAPP ${ }^{* 2,3}$, Jürgen SOTROP ${ }^{* 2}$, Heinz P. HUBER ${ }^{* 2}$, Michael SCHMIDT ${ }^{* 3,4,5}$ \\ ${ }^{*}$ Josef Ressel Center for material processing with ultrashort pulsed lasers, Research Center for \\ Microtechnology, Vorarlberg University of Applied Sciences, Hochschulstraße 1, 6850 Dornbirn, \\ Austria \\ E-mail: matthias.domke@fhv.at \\ ${ }^{* 2}$ Munich University of Applied Sciences, Lothstrasse 34, 80335 Munich, Germany \\ ${ }^{*}$ Graduate School in Advanced Optical Technologies (SAOT), Friedrich-Alexander-Universität \\ Erlangen-Nürnberg, Germany \\ ${ }^{* 4}$ Bayerisches Laserzentrum GmbH, Konrad-Zuse-Str. 2-6, 91052 Erlangen, Germany; \\ ${ }^{*}$ Chair of Photonic Technologies, Friedrich-Alexander-Universität Erlangen-Nürnberg, Germany
}

\begin{abstract}
The selective laser structuring of thin molybdenum films from the glass substrate side using ultra-short laser pulses displays a by up to a factor of 10 higher ablation efficiency compared to the direct laser ablation from the film side. Moreover, holes can be created without burrs, melt formations or micro cracks. A recent simulation of the glass side ablation process, also known as confined laser ablation, suggests that the laser-matter interaction in the molybdenum leads to a confined ultrafast thermal expansion during heating and melting, which generates a pressure wave that causes the metal film to bulge. However, this model disagrees with the general understanding that the confinement of an expanding gas is the driving ablation mechanism. In order to verify this new model, a pump-probe microscopy setup is utilized to investigate the propagation of a pressure wave in the glass substrate. The results show that pressure waves can be detected already at fluences that are not sufficient for evaporation and that they are generated during heating and melting. These observations support the model of ultrafast thermal expansion during heating and melting generating a pressure wave that causes the metal film to bulge.
\end{abstract}

DOI: $10.2961 /$ jlmn.2015.02.0002

Keywords: Selective laser structuring, laser ablation, confined laser ablation, thin film, pump-probe, molybdenum, pressure wave, induced laser ablation

\section{Introduction}

In recent years, several applications for the selective laser structuring by laser ablation of thin metal films by irradiation from the film or substrate side have been discovered, such as direct laser ablation $[1 ; 2]$, the laser induced forward transfer (LIFT) [3], the launch of a laser-driven flyer plate [4], the laser lift-off [5], or laser shock processing [6]. In the case of selective laser structuring of about $430 \mathrm{~nm}$ thin molybdenum films, e.g. for scribing the p-contact of a CIS (Copper-Indium-Diselenide) thin film solar cells, the irradiation of the metal film from the substrate side (Fig. 1 (c)) with ultra-short laser pulses, displays a by up to a factor of 10 higher ablation efficiency compared to the direct laser ablation from the film side (Fig. 1 (a)) [7]. Moreover, holes can be created without burrs, melt formations, or micro cracks. In reference [7] the substrate side laser structuring with 10 ps pulses was referred to as "direct induced laser ablation", which belongs to the group of "confined laser ablation" effects.

The driving mechanisms of the efficient and precise ablation process, is usually adapted from the models for the LIFT [8] and laser shock processing [6]. In both cases, the laser irradiation of the metal film from the glass substrate side generates a confined gas or plasma at the metal-glass interface. It is also assumed for the glass side ablation of thin molybdenum films that the expansion of the gas or plasma causes the metal film to bulge [9-11]. However, this model is not compatible to line scribing with overlap- ping pulses, because the gas could escape at the sides. In fact, structures with arbitrary shape, sharp edges and any pulse to pulse overlap can be produced [12].

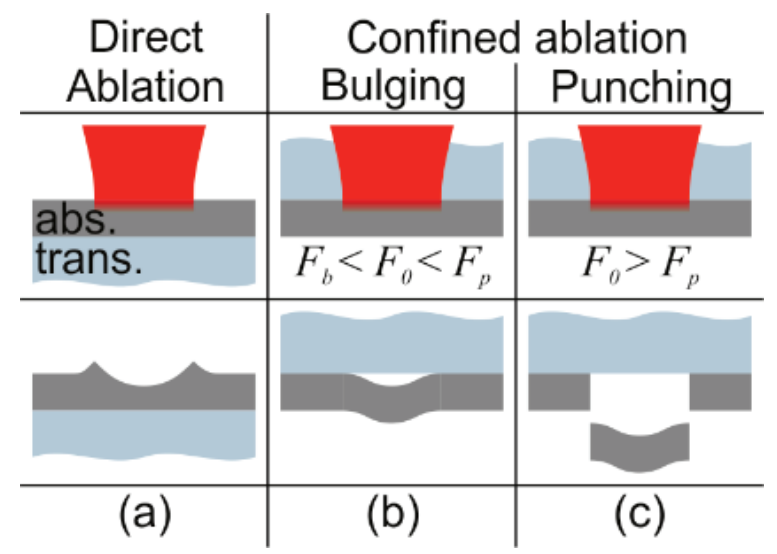

Fig. 1 Ablation effects after laser irradiation of an absorbing layer (grey, abs.) from the film side (a) and from the glass substrate side (b) and (c). Case (a) is called direct laser ablation. In case (b) and (c), the laser pulse transmits through the transparent substrate (blue, trans.), the pulse is then absorbed at interface, and causes a confined laser ablation (also known as "direct induced laser ablation" [7]). The threshold fluence for the bulging of the film in case (b) is called bulging threshold $\mathrm{Fb}$, and for the metal disk lift-off in case (c) it is called punching threshold fluence Fp. 
In order to explain these observations, a numerical simulation was performed by Sotrop et al. [13]. The simulation suggests that an ultrafast thermal expansion during heating and melting - and not a gas expansion-generate a pressure wave, which causes the metal film to bulge. However, this model disagrees completely with the general understanding of the glass substrate side ablation of thin metal films. For this reason, the aim of this study is to verify this model and to better understand the role of pressure waves in confined laser ablation of $430 \mathrm{~nm}$ thin molybdenum films. Time-resolved microscopy is chosen as method to investigate the propagation of the laser-induced pressure waves.

\section{Material and Methods}

\subsection{Pump-Probe Microscopy Setup}

An ultrafast laser source (Spectra-Physics, Spirit), which emits 660 fs (FWHM) pulses at a centre wavelength of $1053 \mathrm{~nm}$, is divided into pump and probe pulse by beam splitter 1 behind the laser exit. The pump-pulse, which is used for initiating the ablation, is focused by a $\mathrm{f}=150 \mathrm{~mm}$ lens on the sample to a spot with a diameter of about $40 \mu \mathrm{m}$ at the $1 / \mathrm{e}^{2}$ intensity level. All given fluence values correspond to the peak fluence of a Gaussian fluence profile without reflectivity correction. The probe-pulse, used for illuminating the sample, is frequency doubled ( $\mathrm{SHG}$ ) to a wavelength of $527 \mathrm{~nm}$ with a pulse duration of about $510 \mathrm{fs}$ (FWHM). Subsequently, a linear translation stage optically delays the probe-pulse up to $4 \mathrm{~ns}$. The sample is coaxially illuminated and imaged by a $20 \mathrm{x}$ microscope objective to a CCD camera. The relative reflectivity change $\Delta R / R=\left(R_{t}-R_{0}\right) / R_{0}$ is measured in the centre of the irradiated spot, where $R_{0}$ is the reflectivity of the original sample and $R_{t}$ is the reflectivity at the pump-probe delay time $\mathrm{t}$. The relative reflectivity change is averaged over an area of $5 \times 5$ pixels which corresponds to a region of $1.6 \times 1.6$ $\mu \mathrm{m}^{2}$. A more detailed description of the pump-probe microscopy setup is given in [14]. A simple diagram of the setup is presented in Fig. 2.

The samples were provided by Plansee. A $430 \mathrm{~nm}$ thin molybdenum film was sputtered on a $1 \mathrm{~mm}$ thick soda-lime glass substrate.

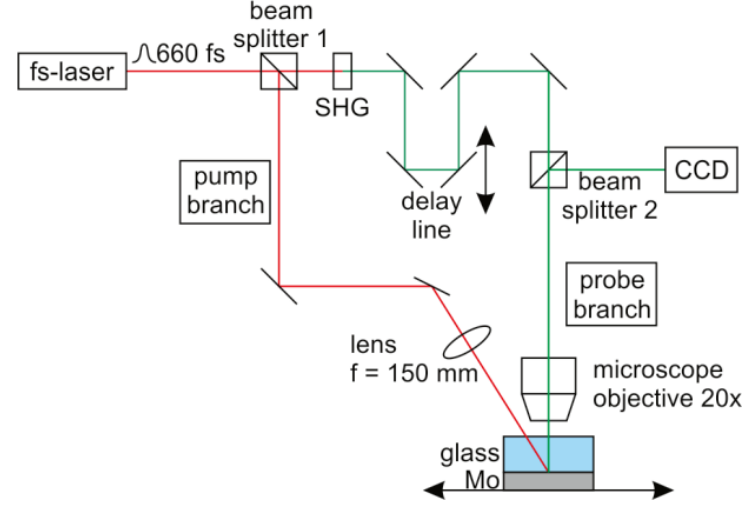

Fig. 2 Pump-probe microscopy setup. The 660 fs pump-pulse (red branch) is focused through the glass substrate on the Mo layer to initiate the ablation. The probe-pulse (green branch) is frequency doubled and delayed on a linear stage to illuminate the sample, which is imaged by a microscope and CCD camera, at different delay times.

\subsection{Model function of the reflectivity signal for the propagation of a laser-induced pressure wave in glass}

According to the simulation in [13], the glass side irradiation of a molybdenum film leads to a temporal and spatial confinement of the laser pulse energy within a few picoseconds and a few tens of nanometers, causing ultrafast thermal expansion of the laser-matter interaction zone. A pressure wave is thus induced in the metal film and in the glass substrate. The pressure wave in the glass substrate can be considered as an infinitely thin layer with increased density. The increased refractive index then leads to Fresnel reflection at the pressure wave front. The reflectivity of the sample from the glass side $R_{t}$ is than influenced by interference of the reflected light from the laser-matter interaction zone with the reflected light from the moving pressure wave front with the reflectivities $R_{1}$ and $R_{2}$, respectively, as sketched in Fig. 3. The phase shift between both reflected wave fronts depends on the distance $d$ of the pressure wave and the refractive index of the glass substrate of $n_{g}=1.5$. Since the pressure wave moves with a velocity $v$, the distance $d=v t$ increases with time, and oscillations appear in the reflectivity signal. Destructive interference can be observed, if the pressure wave has traveled the distance $d=n_{g} \lambda / 4$. The transient reflectivity calculates to

$$
R_{t}=R_{1}+R_{2}+2 \sqrt{R_{1} R_{2}} \cos \left(\frac{4 \pi d n_{g}}{\lambda}\right),
$$

where $\lambda=527 \mathrm{~nm}$ is the vacuum wavelength of the probe pulse.

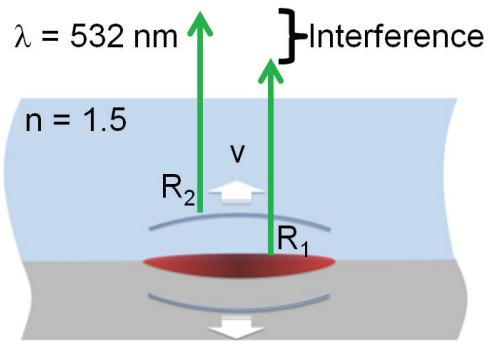

Fig. 3 The laser-induced ultrafast thermal expansion at the molybdenum-glass interface generates pressure waves in the transparent and in the absorbing layers. The pressure wave can be regarded as a moving interface with the reflectivity R2 that propagates towards the probe-pulse with the velocity $\mathrm{v}$. The distance of this interface determines the phase shift between the reflected wave fronts from the pressure wave (R2) and the laser-matter interaction zone (R1). The interference of both reflections lead to an oscillation in the time-resolved reflectivity signal.

Since the reflectivity of the laser-matter interaction zone $R_{1}$ changes with time [11], it is described by a timedependent model function given in equation (2). The quasiinstantaneous excitation of the electrons is described by the error function [15]. The excitation time $\tau_{\mathrm{e}}=840 \mathrm{fs}$ is governed by the cross correlation time of the pump- and probepulse [14]. The time constant $t_{0}$ then describes delay time zero - the time of maximum intensity of the laser pulse. The relaxation of the electrons [16] and the generation of the liquid-gas mixture [17] lead to a reflectivity decrease which can be described as a falling exponential function with the relaxation time $\tau_{r}$ and the initial value $R_{r}$. The con- 
finement leads to a suppression of the expansion of the liquid-gas mixture, and the subsequent thermal diffusion and condensation is expressed by summing a second exponential function with the time constant $\tau_{d}$ and the initial value $R_{d}$. Melting and damaging of the interface leads to a permanent reflectivity change, which is described by the final value $R_{\infty}$. The transient reflectivity of the laser-matter interaction zone is thus written as

$$
R_{1}=\frac{1}{2}\left(1+\operatorname{erf}\left(\frac{t-t_{0}}{\tau_{e}}\right)\right)\left(R_{r} e^{-\frac{t-t_{0}}{\tau_{r}}}+R_{d} e^{-\frac{t-t_{0}}{\tau_{d}}}+R_{\infty}\right)
$$

The pressure wave in the glass causes the second interface with the reflection $R_{2}$ in the interference equation (1). The model by Sotrop et al. suggests that the pressure wave is generated already during the excitation of the electrons [13]. This process is therefore described also by the error function. The reflectivity at the pressure wave $R_{p w}$ is approximated to be constant. The transient reflectivity of the pressure wave is thus written as

$$
R_{2}=R_{p w} \frac{1}{2}\left(1+\operatorname{erf}\left(\frac{t-t_{0}}{\tau_{e}}\right)\right)
$$

Finally, the velocity of the pressure wave determines the period of the oscillation. The velocity of a pressure wave in quartz glass is - in contrast to air - constant up to a pressure of about $20 \mathrm{GPa}$ [18] and in soda-lime glass it is constant at least up to $6 \mathrm{GPa}$ [19]. The released pressure in the glass substrate for a confined laser ablation can be estimated as follows. The spallation strength of Mo is about $3 \mathrm{GPa}$ [20] and should approximately correspond to the pressure in the molybdenum at the ablation threshold fluence. The comparison of the acoustic impedances of Mo and soda-lime glass suggests that only about $25 \%$ of the pressure is transmitted into the glass. The velocity of the pressure wave can thus be assumed to remain constant in the glass, even at fluences well above the ablation threshold.

\section{Results and Discussion}

\subsection{Pressure waves below the bulging threshold}

The transient reflectivity change of a molybdenum film, which is observed from the substrate side and irradiated with a fluence below the bulging threshold of about $F_{b}<0.3 \mathrm{~J} / \mathrm{cm}^{2}$ from the same side, is shown in Fig. 4. In this fluence regime, the irradiation does not cause any mechanical deformation in the molybdenum film. At a fluence of $F_{0}=0.08 \mathrm{~J} / \mathrm{cm}^{2}$, the relative reflectivity increases by $5 \%$ within $1 \mathrm{ps}$ around $t=0 \mathrm{ps}$ and then decreases to $0 \%$ after 50 ps. However, pressure-wave-induced oscillations cannot be distinguished from the noise level in the reflectivity signal. If the fluence is increased to $F_{0}=0.13 \mathrm{~J} / \mathrm{cm}^{2}$, the reflectivity increases by $8 \%$ within 1 ps and then decreases to $0 \%$ after $100 \mathrm{ps}$. Now, an oscillation can be clearly identified in the signal, especially in the time frame between $100 \mathrm{ps}$ and $200 \mathrm{ps}$. If the fluence is increased further to $F_{0}=0.23 \mathrm{~J} / \mathrm{cm}^{2}$, the steep reflectivity increase around $t=0 \mathrm{ps}$ remains at the same level. However, the amplitude of the oscillation increases further with the fluence, but the phase and period remain constant. The period of the oscillation is about $30 \mathrm{ps}$, with maxima at about $30 \mathrm{ps,} 60 \mathrm{ps}$, and 90 ps. It was found that this period remains also constant, if the film or substrate thickness, the type of metal film, or the pulse duration is changed.

The observation of oscillations in the reflectivity signal in the fluence regime below the bulging threshold $F_{0}<F_{b}$ indicates that a pressure wave is already induced in the glass substrate, even though the film has not been set into motion. Moreover, these low fluences are not sufficient for material ablation, if molybdenum is irradiated directly from the film side (Fig. 1 case (a)) [11]. If the different reflectivities for film and glass side irradiation are taken into account, the applied fluences in this regime are even insufficient to generate a spatially confined partial evaporation of the Mo film by glass side irradiation. The state of matter of the laser-matter interaction zone can be also estimated from the reflectivity transients. It has been shown in several time-resolved reflectivity measurements that the formation of a liquid-gas mixture leads to scattering and absorption of the probe pulse, and thus to a steep negative reflectivity change [17]. This is also observed at higher fluences in Fig. 5. However, the reflectivity change in Fig. 4 remains positive for all fluences in the whole time frame. These observations indicate that the measured pressure wave cannot be induced by the expansion of a confined gas. These findings are in good agreement with the simulations and the model of Sotrop et al. [13]. The results of the simulation suggest that the ultrafast thermal expansion of the laser-matter interaction zone during heating and melting already initiates pressure waves in the film and substrate. Moreover, it was calculated that evaporation does not take place in the fluence regime below the measured bulging threshold. Another finding of the simulation is the temporal sequence of melting and evaporation; melting occurs at about 1 ps to $5 \mathrm{ps}$ and evaporation at about $50 \mathrm{ps}$. The measurements clearly show that the pressure wave starts around or a few ps after delay time zero-which is the temporal start of melting according to the simulations of Sotrop et al. [13].

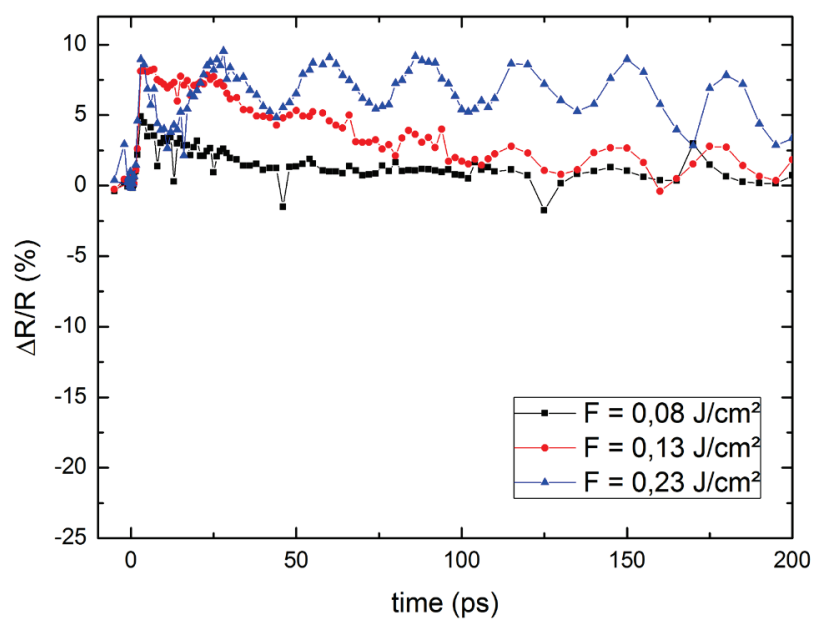

Fig. 4 Transient reflectivity change $\Delta R / R$ of a molybdenum film, which is irradiated with fluences below the bulging threshold of $F_{b}<0.3 \mathrm{~J} / \mathrm{cm}^{2}$ and was also measured from the glass substrate side. The observation of oscillations and their temporal start at sub-threshold conditions indicate that a pressure wave is already generated by ultrafast expansion during heating and melting of the confined laser-matter interaction zone. 


\subsection{Pressure waves above the bulging threshold}

Now, the transient relative reflectivity change is considered at fluences above the bulging threshold fluence (Fig. 1 (b)) for $F_{0}=0.3 \mathrm{~J} / \mathrm{cm}^{2}$ and $F_{0}=0.45 \mathrm{~J} / \mathrm{cm}^{2}$ and above the punching threshold fluence (Fig. 1 (c)) for $F_{0}=0.63 \mathrm{~J} / \mathrm{cm}^{2}$ in Fig. 5. The transient behavior is comparable for all fluences, however, on a temporal average the amplitudes of the relative reflectivity change decrease with the fluence. For all fluences, the reflectivity increases to a first maximum within a few ps after delay time zero, and then drops to a minimum at about $10 \mathrm{ps}$. The relative reflectivity change at the minimum has values of $-1 \%,-12 \%$, and $-23 \%$ for $0.30 \mathrm{~J} / \mathrm{cm}^{2}, 0.45 \mathrm{~J} / \mathrm{cm}^{2}$, and $0.63 \mathrm{~J} / \mathrm{cm}^{2}$, respectively. Subsequently, an oscillation with a constant period of about 30 ps can be observed for all fluences, with maxima at about $30 \mathrm{ps}, 60 \mathrm{ps}$, and $90 \mathrm{ps}$, while the average reflectivity change increases slightly with time. For comparison, Fig. 5 also shows the relative reflectivity change for the film side (FS) irradiation of molybdenum at a fluence of $0.6 \mathrm{~J} / \mathrm{cm}^{2}$ in green. It can be seen that the reflectivity increases up to $7 \%$ shortly after $\mathrm{t}=0 \mathrm{ps}$, then decreases exponentially to about $-80 \%$ (See inset in Fig. 5). However, the slope of the relative reflectivity change is not as steep as for glass substrate side irradiation.

Fig. 5 also contains the fit of the interference equation (1) to the measured data points for glass side irradiation. The velocity, with which the pressure wave propagates through the glass, was determined to be $\mathrm{v}=5910(34) \mathrm{m} / \mathrm{s}$ at $F_{0}=0.33 \mathrm{~J} / \mathrm{cm}^{2}, \mathrm{v}=5903(38) \mathrm{m} / \mathrm{s}$ at $F_{0}=0.45 \mathrm{~J} / \mathrm{cm}^{2}$, and $\mathrm{v}=5984(36) \mathrm{m} / \mathrm{s}$ at $F_{0}=0.63 \mathrm{~J} / \mathrm{cm}^{2}$. These velocities agree very well to the sound velocity of soda-lime glass of about $5840 \mathrm{~m} / \mathrm{s}$ [19]. The amplitude of the measured data points with respect to the fit curve is higher below and lower above $100 \mathrm{ps}$. This deviation could be explained by a damping of the pressure wave, which was not taken into account in equation (3) in order to keep the parameter space as small as possible. The decay times are determined to be $\tau_{r}=2-3 \mathrm{ps}\left(0.33 \mathrm{~J} / \mathrm{cm}^{2}, 0.45 \mathrm{~J} / \mathrm{cm}^{2}\right.$ and $\left.0.63 \mathrm{~J} / \mathrm{cm}^{2}\right)$ and $\tau_{d}=50-60 \mathrm{ps} \quad\left(0.33 \mathrm{~J} / \mathrm{cm}^{2}\right.$ and $\left.0.63 \mathrm{~J} / \mathrm{cm}^{2}\right)$. For $0.45 \mathrm{~J} / \mathrm{cm}^{2}$, the decay time $\tau_{d}$ is about $500 \mathrm{ps}$. The decay time $\tau_{r}$ describes the steep reflectivity decrease from positive to negative values between $0 \mathrm{ps}$ and $10 \mathrm{ps}$, and the decay time $\tau_{d}$ describes the subsequent slight reflectivity increase up to $200 \mathrm{ps}$. The strong variation of the fit values for $\tau_{d}$ can be explained as follows. The model function only describes the signal up to a delay time of $t_{d s}=2 d_{s} / c_{s} \approx 150 \mathrm{ps}$. During this time frame, the pressure wave propagates through the film with the thickness $d_{s}=430 \mathrm{~nm}$ at the sound velocity of about $6000 \mathrm{~m} / \mathrm{s}$, it is being reflected at the free surface, and finally returns to the molybdenum-glass interface. Then, the superposition of two pressure waves [21] or the delamination of the film [13] can suddenly change the reflectivity trend. This can influence the second decay time, as observed for $0.45 \mathrm{~J} / \mathrm{cm}^{2}$. A more complex model is thus necessary to describe the reflectivity at longer delay times.

The comparison of the reflectivity signal of film and substrate side irradiation at fluences of $0.6 \mathrm{~J} / \mathrm{cm}^{2}$ (Fig. 5 , blue squares) and $0.63 \mathrm{~J} / \mathrm{cm}^{2}$ (Fig. 5, green triangles), respectively, shows interesting features during the formation of a gas-liquid mixture occurring at twice the bulging threshold fluence, namely a steep drop of reflection by scattering and absorption of the probe pulse [17]. The relative reflectivity change for glass substrate side irradiation decreases faster, it reaches a minimum of about $-23 \%$ at about $10 \mathrm{ps}$, and afterwards it increases with time. The appearance of a reflectivity minimum at $10 \mathrm{ps}$ can be explained by the confinement of the laser-matter interaction zone, which inhibits the expansion of a liquid-gas mixture. The decay time $\tau_{r}$, which is about $2-3 \mathrm{ps}$, agrees here very well to the typical melting time of metals such as e.g. aluminum [22]. The pressure wave thus should be generated within this time frame, when melting and not evaporation is expected. This is in good agreement with the simulation, which shows that the melting takes about $5 \mathrm{ps}$ and the evaporation about $10-20 \mathrm{ps}$ [13]. The subsequent reflectivity increase for delay times of $50 \mathrm{ps}$ and higher could be caused by heat diffusion and condensation of the confined liquid-gas mixture. In contrary, the film side reflectivity change starting from about $10-20$ ps continues to drop to a value of $\Delta R / R=-75 \%$ within the observation time, indicating a strongly scattering and absorbing gas-liquid mixture expanding from the film side into the half space [17].

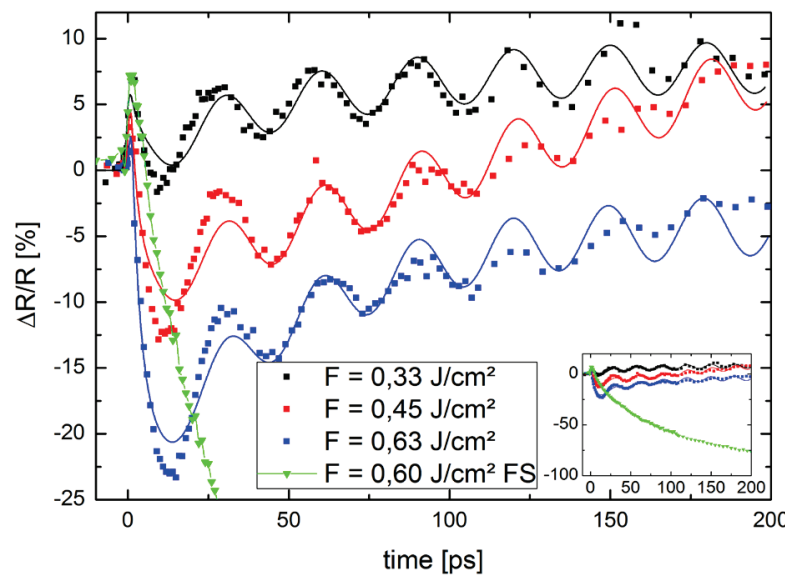

Fig. 5 Transient reflectivity change $\Delta R / R$ of a molybdenum film, which is irradiated with fluences above the bulging threshold of $\mathrm{Fb}>0.3 \mathrm{~J} / \mathrm{cm}^{2}$, and is also measured from the glass substrate side. The solid lines represent the fit of equation (1) to the data points. The green triangles represent the transient reflectivity change for film side (FS) irradiation. The inset shows the data points in the same time frame but with an enhanced y-axis. The steeper decrease of reflectivity during substrate side irradiation in comparison to film side irradiation indicates that a pressure wave must be already generated by the ultrafast expansion during heating and melting of the confined laser-matter interaction zone.

The comparison of the reflectivity signal at fluences below and above the bulging threshold should now reveal the influence of the formation of a gas-liquid mixture at the interface on the pressure wave propagation in glass. This comparison indicates that the period and the phase of the oscillation for below and above threshold displays the same properties (the amplitude increases only slightly with the fluence, the signal is shifted downwards on the y-axis with increasing fluence, but the signal trend shows no anomalous behavior). Consequently, there is no indication for a fundamental change to the pressure wave, originated 
from the expansion of gas at fluences above the bulging and punching threshold. Thus, the pressure wave in the glass is initiated during heating and melting at fluences below as well as above the bulging threshold. These findings further suggest that the delamination of the film is rather caused if the pressure of the pressure wave exceeds the adhesion force of the film, then by the adiabatic expansion of confined gas.

\section{Conclusion}

The general understanding of the glass substrate side ablation of thin molybdenum films with ultrashort pulse lasers is based on the assumption that a confined gas is generated during the laser-matter interaction at the molybdenum-glass interface, and that the expansion of the gas causes the film to bulge. However, a recent simulation suggests that the ultrafast thermal expansion during heating and melting initiates a pressure wave, which causes the metal film to bulge.

In order to verify this new model, the pressure wave generation and propagation in the glass substrate was investigated using pump-probe microscopy. The interference of the probe light reflections from the pressure wave front and from the molybdenum/glass interface enable an interferometric measurement of the pressure wave propagation in the glass substrate.

Pressure waves were detected already at fluences below the bulging threshold. Since the fluence in this regime is not sufficient for evaporation, these findings suggest that the observed pressure wave was generated by the ultrafast thermal expansion during heating and melting. At fluences above the bulging and punching threshold, the simulation by Sotrop et al. suggested that heating and melting takes place within $5 \mathrm{ps}$ and evaporation after $10 \mathrm{ps}$ to $20 \mathrm{ps}$. However, the measurements show that the temporal start point of the pressure wave remains at higher fluences also around delay time zero. That is the time frame, when only heating and ultrafast melting occurs. Moreover the oscillations are not changed in the time frame when evaporation is expected by the simulation of Sotrop et al. Those observations suggest that the ultrafast thermal expansion during heating and melting generates a pressure wave, which causes the metal film to bulge. However, the expansion of a confined gas seems to have no contribution to the bulging of the film, as suggested by the simulation of Sotrop et al.

\section{Acknowledgements}

Christian Linke from Plansee is acknowledged for providing the samples. The authors gratefully acknowledge funding of the Erlangen Graduate School in Advanced Optical Technologies (SAOT) by the German Research Foundation (DFG) in the framework of the German excellence initiative. This work was partly funded by the Seventh Framework Programme of the European Commission within the project 'Solardesign', under Grant No. 310220. Spectra Physics is acknowledged for the close collaboration and the financial support of the Josef Ressel Center for material processing with ultrashort pulsed lasers. The financial support by the Austrian Federal Ministry of Economy, Family and Youth and the National Foundation for
Research, Technology and Development is gratefully acknowledged.

\section{References}

[1] Zoppel, S.; Huber, H.; Reider, G. A. Applied Physics A: Materials Science \& Processing. 89. 1 (2007).

[2] Compaan, A. D.; Matulionis, I.; Nakade, S. Optics and Lasers in Engineering. 34. 1 (2000).

[3] Bohandy, J.; Kim, B. F.; Adrian, F. J. Journal of Applied Physics. 60. 4 (1986).

[4] Watson, S.; Field, J. E. Journal of Physics D: Applied Physics. 33. 2 (2000).

[5] Wong, W. S.; Sands, T.; Cheung, N. W. Applied Physics Letters. 72.5 (1998).

[6] Fabbro, R.; Fournier, J.; Ballard, P.; Devaux, D.; Virmont, J. Journal of Applied Physics. 68. 2 (1990).

[7] Heise, G.; Englmaier, M.; Hellwig, C.; Kuznicki, T.; Sarrach, S.; Huber, H. Applied Physics A: Materials Science and Processing. 102. 1 (2011).

[8] Adrian, F. J.; Bohandy, J.; Kim, B. F.; Jette, A. N.; Thompson, P. J Vac Sci \& Techn B. 5.5 (1987).

[9] Heise, G.; Domke, M.; Konrad, J.; Sarrach, S.; Sotrop, J.; Huber, H. P. Journal of Physics D: Applied Physics. 45. 31 (2012).

[10]Bartl, D.; Michalowski, A.; Hafner, M.; Letsch, A.; Nolte, S.; Tünnermann, A. Appl Phys A. 110. 1 (2013).

[11]Domke, M.; Rapp, S.; Schmidt, M.; Huber, H. P. Applied Physics A: Materials Science and Processing. 109. 2 (2012).

[12]Huber, H. P.; Englmaier, M.; Hellwig, C.; Heiss, A.; Kuznicki, T.; Kemnitzer, M.; Vogt, H.; Brenning, R.; Palm, J. Proceedings of SPIE - The International Society for Optical Engineering. 7203 (2009).

[13]Sotrop, J.; Kersch, A.; Domke, M.; Heise, G.; Huber, H. P. Applied Physics A. 113. 2 (2013).

[14]Domke, M.; Rapp, S.; Schmidt, M.; Huber, H. P. Opt Express. 20. 9 (2012).

[15]Holzapfel, W.; Finkele, U.; Kaiser, W.; Oesterhelt, D.; Scheer, H.; Stilz, H. U.; Zinth, W. Proceedings of the National Academy of Sciences of the United States of America. 87. 13 (1990).

[16]Hohlfeld, J.; Wellershoff, S. S.; Güdde, J.; Conrad, U.; Jähnke, V.; Matthias, E. Chemical Physics. 251. 1-3 (2000).

[17]Von Der Linde, D.; Sokolowski-Tinten, K. Applied Surface Science. 154-155 (2000).

[18]Marsh, S. P. LASL shock hugoniot data (University of California Press, 1980).

[19]Millett, J.; Bourne, N.; Rosenberg, Z. Journal of Applied Physics. 84. 2 (1998).

[20]Duffy, T. S.; Ahrens, T. J. Journal of Applied Physics. 76. 2 (1994).

[21]Wellershoff, S.-S. Untersuchungen zur Energierelaxationsdynamik in Metallen nach Anregung mit ultrakurzen Laserpulsen (Dissertation.de, 2000).

[22]Kandyla, M.; Shih, T.; Mazur, E. Physical Review B. 75. 21 (2007).

(Received: June 18, 2014, Accepted: January 28, 2015) 